\title{
Placebo controlled pilot trial to study the remyelinating potential of intravenous immunoglobulins in multiple sclerosis
}

\author{
Martin Stangel, Friedrich Boegner, Christel H Klatt, Christoph Hofmeister, Sepp Seyfert
}

Department of

Neurology,

Universitätsklinikum

Benjamin Franklin,

Freie Universität

Berlin,

Hindenburgdamm 30, D-12200 Berlin,

Germany

M Stangel

F Boegner

C Hofmeister

S Seyfert

Clinical

Neurophysiology

C H Klatt

Correspondence to:

Dr M Stangel, Department

of Neurology,

Universitätsklinikum

Benjamin Franklin,

Hindenburgdamm 30,

D-12200 Berlin, Germany

email

mstangel@zedat.fu-berlin.de

Received 1 June 1999 and in revised form

16 August 1999

Accepted 3 September 1999

\begin{abstract}
Currently there is no treatment available to improve a stable deficit in multiple sclerosis. It was shown in animal models that intravenous immunoglobulins (IVIg) can enhance central nervous remyelination, and the first open trials were promising. We therefore conducted a double blind, placebo controlled pilot study to evaluate the effect of IVIg treatment in patients with multiple sclerosis with a stable clinical deficit. The primary outcome parameter was the change in central motor conduction time as an indirect measure of central myelination. Secondary outcome parameters were neurological examinations including the expanded disability status scale (EDSS), neurological rating scale (NRS), and manual muscle testing (MMT). Ten patients were treated first with placebo and then with IVIg $(0.4 \mathrm{~g} / \mathrm{kg}$ body weight on 5 consecutive days), the two treatments being separated by an interval of 6 weeks. There was no difference in the central motor conduction times measured before and 6 weeks after each treatment. Clinically there was a small improvement after IVIg treatment, but there was no significant difference when compared with placebo. In conclusion, our data do not support a role for IVIg in the remyelination of stable multiple sclerosis lesions as measured by central conduction time. The importance of the small clinical benefit is currently not clear.

(F Neurol Neurosurg Psychiatry 2000;68:89-92)
\end{abstract}

Keywords: multiple sclerosis; intravenous immunoglobulins; remyelination

Multiple sclerosis is a disabling disease characterised by demyelination of the CNS). There is currently no therapy to improve a persistent clinical deficit. Spontaneous remyelination occurs in multiple sclerosis lesions, but it is often incomplete. ${ }^{12}$ Augmentation of such remyelination could provide repair of lesions and possibly reverse a neurological impairment. Treatment with intravenous immunoglobulins (IVIg) modulates the immune sys- tem and has been used successfully in some autoimmune neurological disorders. ${ }^{3}$ In multiple sclerosis, administration of IVIg can reduce the relapse rate and the number of gadolinium enhancing lesions on MRI. ${ }^{4-6}$ In an experimental model of multiple sclerosis-Theiler's virus encephalomyelitis - it was demonstrated that immunoglobulins can enhance remyelination. ${ }^{78}$ In small open trials, visual acuity and colour vision improved in stable optic neuritis in patients with multiple sclerosis after IVIg treatment, ${ }^{9}$ and there was clinical improvement of paresis. ${ }^{10}$ We have conducted a pilot trial investigating the remyelinating potential of IVIg using central conduction times as a measure of CNS myelination.

\section{Patients and methods}

PATIENTS

Inclusion criteria were clinically definite relapsing-remitting multiple sclerosis ${ }^{11}$ with an expanded disability status scale (EDSS) ${ }^{12}$ score between 2.0 and 4.5, and a clinically stable neurological deficit. The last clinical relapse must have occurred more than 3 months ago without an ongoing improvement. Exclusion criteria were treatment with interferon- $\beta$ or other immunomodulatory therapies, pregnancy, diabetes mellitus, renal insufficiency, liver disease, and heart disease.

All patients gave written informed consent before inclusion, and the study has been approved by the local ethics committee.

\section{STUDY DESIGN}

The study was designed as a double blind placebo controlled trial. A 6 week run in period to document the stability of the clinical deficit preceded two identical and consecutive 6 week treatment periods. Based on previous trials ${ }^{913}$ we chose a time span of 6 weeks for treatment evaluations. Each patient was treated first with placebo and then with IVIg to act as his or her own control. This non-randomised design was chosen to avoid a carry over effect due to the long half life of IVIg. A much larger sample size would have been required for the comparison in a two group analysis, ${ }^{13}$ and a longer wash out period would increase the chance of relapses that would interfere with the study. Patients, evaluating neurologist, electrophysiological 
Table 1 Baseline characteristics for the enrolled patients

\begin{tabular}{lllllll}
\hline Patient No & Age & Sex & $\begin{array}{l}\text { Duration of } \\
\text { MS }(y)\end{array}$ & $\begin{array}{l}\text { Last relapse } \\
\text { (months) }\end{array}$ & $\begin{array}{l}\text { Baseline } \\
\text { EDSS }\end{array}$ & $\begin{array}{l}\text { Baseline } \\
\text { NRS }\end{array}$ \\
\hline 1 & 38 & M & 10 & 24 & 3,5 & 71 \\
2 & 46 & M & 25 & 4 & 4,0 & 71 \\
3 & 49 & M & 10 & 4 & 3,0 & 92 \\
4 & 54 & F & 13 & 9 & 4,0 & 78 \\
5 & 53 & F & 27 & 11 & 3,5 & 84 \\
6 & 28 & M & 8 & 4 & 4,0 & 60 \\
7 & 38 & M & 12 & 4 & 4,0 & 60 \\
8 & 38 & M & 6 & 4 & 3,5 & 75 \\
9 & 31 & F & 6 & 14 & 3,5 & 67 \\
10 & 55 & F & 12 & 11 & 2,0 & 81 \\
Mean & 43 & $6 \mathrm{M} / 4 \mathrm{~F}$ & 12.9 & 8.9 & 3.5 & 73.9 \\
Range & $28-55$ & & $6-27$ & $4-24$ & $2,0-4,0$ & $60-92$ \\
\hline
\end{tabular}

examiner, and nurses were blinded to this treatment regimen.

Patients received $0.4 \mathrm{~g} / \mathrm{kg}$ body weight IVIg ( $5 \%$ IgG in $5 \%$ sorbitol, $0.9 \% \mathrm{NaCl}$, maximum $0.6 \%$ PEG, pH 5-6) on 5 consecutive days. Placebo treatment consisted of the identical amount of the carrier substances, delivered in identical flasks.

The primary outcome measure was change in central motor conduction time. Secondary outcome measures were neurological function tests and neurological scales.

\section{ELECTROPHYSIOLOGICAL STUDIES}

Electrophysiological measurements included standard motor evoked potentials (MEPs) using a Nicolet Viking myograph and a Dantec Mag Pro stimulator with a circular coil type 16 E 57 for individual stimulations. The centre of the coil was held tangentially over Fz (international 10/20 system). Muscle responses were recorded with surface electrodes placed over the $M$ extensor digitorum brevis. The stimulation intensity was $20 \%$ above the threshold, and the preinnervation was kept stable at $10 \%$ of maximal force by analog recording on a separate monitor. The peripheral conduction time was calculated by the formula:

\section{peripheral conduction time $=(\mathrm{F}$ wave latency $+M$ wave latency) $/ 2^{14} 15$}

Central motor conduction was calculated as the total conduction time minus peripheral conduction time with the normal range being $12.5 \mathrm{~ms} \pm 3.4$ (2 SD); with peak to peak amplitude in $\mathrm{mV} .{ }^{16}$ Of a total of 12 measurements the shortest and longest latencies were ignored and values calculated as the mean of the remaining 10 measurements. Electrophysiological measurements were made by the same examiner at inclusion into the study, 3-14 days before the

Table 2 Electrophysiological and clinical measurements

\begin{tabular}{lllll}
\hline & At inclusion & Before placebo & $\begin{array}{l}\text { After placebol } \\
\text { before IVIg }\end{array}$ & After IVIg \\
\hline $\begin{array}{l}\text { MEP (ms): } \\
\quad \text { To left leg }\end{array}$ & $22.78(8.6)$ & $23.14(10.5)$ & $22.77(9.0)$ & $21.71(8.6)$ \\
$\quad \begin{array}{l}\text { To right leg } \\
\text { Amplitude (mV): }\end{array}$ & $23.34(10.4)$ & $24.68(11.7)$ & $23.95(11.2)$ & $23.6(11.3)$ \\
$\quad$ To left leg & $1.51(0.9)$ & $1.45(1.3)$ & $1.46(1.1)$ & $1.44(1.2)$ \\
$\quad$ To right leg & $1.14(0.6)$ & $1.36(0.7)$ & $0.89(0.5)$ & $1.01(0.8)$ \\
EDSS & $3.5(0.6)$ & $3.5(0.6)$ & $3.45(0.9)$ & $3.4(0.6)$ \\
NRS & $73.9(10.3)$ & $71.7(9.1)$ & $74.8(8.4)$ & $78.0(6.8)$ \\
MMT & $226.5(9.9)$ & $227.7(11.9)$ & $228.7(9.0)$ & $232.1(6.0)$ \\
Walk 20 m (s) & $13.9(3.1)$ & $13.8(2.6)$ & $14.4(3.4)$ & $13.5(2.3)$ \\
Climb 12 steps (s) & $7.3(1.3)$ & $7.3(1.3)$ & $7.3(1.3)$ & $7.2(2.1)$ \\
\hline
\end{tabular}

Values in parentheses are SD. first treatment, 6 weeks after the first treatment (which was 1-5 days before the second treatment), and 6 weeks after the second treatment.

NEUROLOGICAL ASSESSMENT

Each neurological evaluation included EDSS score, Scripps neurological rating scale (NRS), ${ }^{17}$ time to walk $20 \mathrm{~m}$ unaided, time to climb 12 steps, and a manual muscle test (MMT). ${ }^{18}$ Neurological functions were assessed at the same times as the electrophysiological testing.

\section{STATISTICAL ANALYSIS}

Analysis of the primary and secondary outcome measures with repeated measures analysis of variance (ANOVA) were performed. Multivariate tests were used if assumptions for univariate testing was possibly violated. Due to the small sample size results up to $p=0.1$ were considered for post hoc analysis by $t$ test for single measure comparisons. Treatment periods were compared using two way ANOVA with repeated measurements on both factors (before and after treatment and placebo and IVIg effect). Differences in the response to the stimulus between placebo and treatment groups were tested by the interaction effect of this two factorial design.

\section{Results}

All 10 patients included in the study completed all evaluations. Baseline demographic and clinical data for the patients are given in table 1 . There were no major changes of the electrophysiological measurements and the neurological rating during the 6 week run in period from inclusion until the first treatment (table 2), demonstrating that the patients were in a stable clinical condition.

EFFECT OF IVIg ON MEP, THE PRIMARY OUTCOME MEASURE

Measurement of MEPs before and 6 weeks after each of the two treatments showed only minor changes that were not significant. After IVIg treatment, the central conduction time decreased by 0.71 (SD 2.3) ms compared with 0.55 (SD 2.7) ms after placebo (fig). Thus, no evidence was discovered for IVIg affecting central myelination as measured by MEP. F Wave latencies as indicators of peripheral conduction time were likewise unaffected. Similarly, no significant change in amplitudes was found (table 2).

EFFECT OF IVIg ON NEUROLOGICAL FUNCTION There was a significant improvement in the NRS 6 weeks after IVIg treatment $(p=0.029$; table 2). However, there was also an improvement (non-significant, $\mathrm{p}=0.081$ ) after placebo treatment, and comparison of the two treatment periods showed no significant difference. A similar small improvement was seen in the MMT with both treatments. Thus these improvements are likely to represent a placebo effect. No significant changes were found for EDSS, time to walk $20 \mathrm{~m}$, and time to climb 12 steps. 


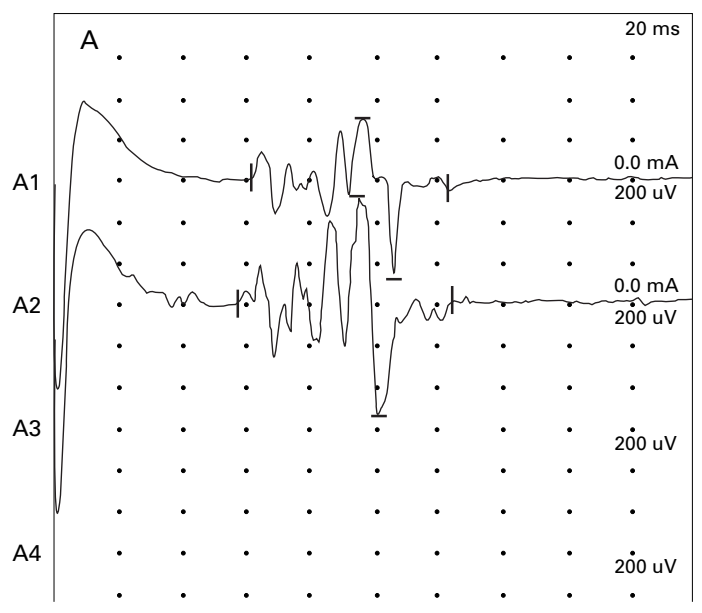

Recording Site :

\begin{tabular}{|l|c|c|c|c|}
\hline \multicolumn{1}{|c|}{ STIMULUS SITE } & $\begin{array}{c}\text { LAT1 } \\
\mathrm{ms}\end{array}$ & $\begin{array}{c}\text { DVR } \\
\mathrm{ms}\end{array}$ & $\begin{array}{c}\text { AMP } \\
\mathrm{mV}\end{array}$ & $\begin{array}{c}\text { AREA } \\
\mathrm{mVms}\end{array}$ \\
\hline A1: & 61.5 & 62.0 & 0.790 & 5.655 \\
A2: & 56.9 & 68.1 & 0.984 & 10.79 \\
A3: & & & & \\
A4: & & & & \\
A5: & & & & \\
\hline
\end{tabular}

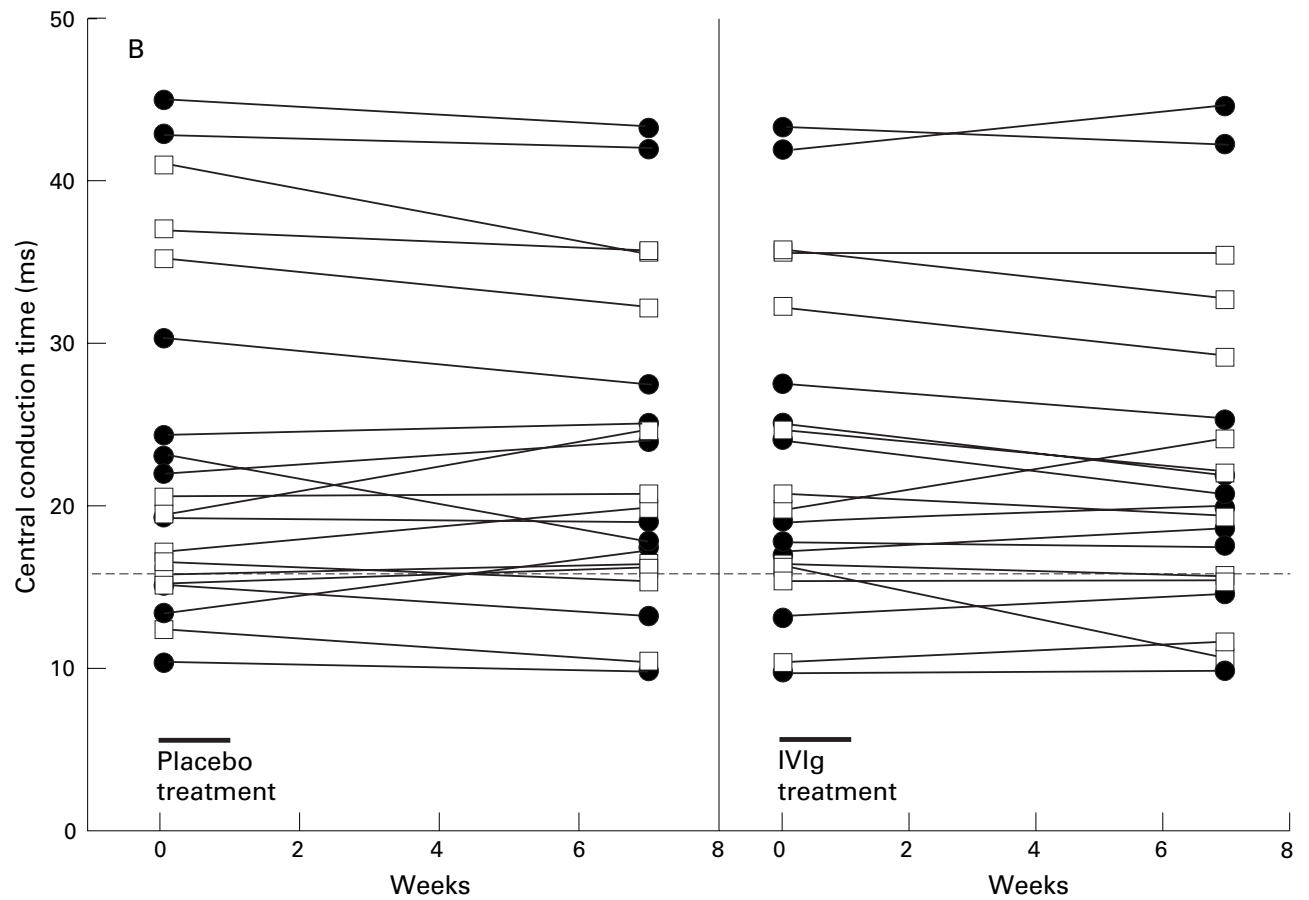

(A) Original track record of a representative magnetic stimulation. (B) Changes in central motor conduction time to both legs as measured by MEP before and 6 weeks after placebo and IVIg treatment, respecitvely. The dotted line indicates the upper limit as $12.5 \mathrm{~ms} \pm 2 \mathrm{SD}(3.4 \mathrm{~ms})$. Filled circles are used for measurements to the right leg, open squares to the left leg.

ADVERSE EFFECTS

Treatment with IVIg was well tolerated. The only adverse effect occurring more often during IVIg treatment was headache (six patients during IVIg treatment, three patients during placebo treatment). Laboratory tests showed the expected rise of total serum protein and IgG concentration from 11.0 (SD 3.1) g/1 to 42.3 (SD 9.1) $\mathrm{g} / \mathrm{l}$. Changes for all other laboratory tests (full blood count, liver enzymes, renal variables, and blood glucose) were only minor.

\section{BLINDING}

After completion of the trial, patients and evaluating neurologist were asked to assign which of the treatments they considered as placebo or IVIg. Four patients guessed correctly, one because of the experienced side effects (headache) during active treatment. One pa- tient guessed incorrectly and five were not able to assign any treatment to IVIg or placebo. The evaluating physician guessed correctly in four patients and incorrectly in four patients and was not able to decide this question in two patients. This suggests that blinding was valid for both patients and evaluating neurologist.

\section{Discussion}

To evaluate the potential of IVIg to induce remyelination in patients with multiple sclerosis with stable motor deficit, this is the first study using as primary outcome central motor conduction as the best available objective measure of CNS myelination. No difference was seen between placebo and active treatment. This is in line with data from another IVIg treatment study in multiple sclerosis with MRI as the main evaluating instrument, in which only a non-significant improvement in 
the central motor conduction was seen during IVIg treatment. ${ }^{6}$

The initial results from an open study with a similar patient population as ours showed some clinical benefit after IVIg, but this study did not include electrophysiological measurements and had no placebo control. ${ }^{10}$ Subsequent controlled trials on stable motor deficit and optic neuritis in multiple sclerosis failed to confirm the positive results. ${ }^{1920}$

There are several possible explanations why remyelination by IVIg could not be demonstrated in our and in other studies: (1) It is not clear how long and what dose of IVIg should be administered to promote remyelination, and possibly our treatment protocol was too short. However, other trials with longer treatments similarly did not show a reversal of a permanent deficit. ${ }^{1920}$ (2) The time of treatment, when there is a fixed deficit, may be too late for a remyelinating therapy, for which there is possibly only a certain yet unknown time window. Another important question is whether IVIg can enter the brain parenchyma to target the remyelinating cells. (3) Studies in the Theiler's virus model have shown that the immunoglobulins most effectively promoting remyelination were IgMs that recognise antigens on oligodendroglial cells. ${ }^{21}$ The IVIg preparations used in multiple sclerosis studies so far contain no or only traces of $\operatorname{IgM}$. (4) Finally, although the central conduction time is currently the best availabe measure of CNS myelination, the degree of clinical deficit in multiple sclerosis and the change in evoked potential latency may correlate poorly. In this respect, the importance of the small clinical benefit seen in our study is currently not clear, but the study was not designed to evaluate this aspect.

Many studies have shown that IVIg is a potent immunomodulator ${ }^{3223}$ and there is compelling evidence that IVIg has a beneficial effect on relapse rate in multiple sclerosis. Despite promising results from experimental work and clinical pilot trials, this and other studies were thus far unable to demonstrate an effect of IVIg on myelin repair in permanent defects in multiple sclerosis.

The study was supported by Grifols Deutschland GmbH, Langen, Germany, providing IVIg and placebo. MS was the recipient of a DFG research scholarship during part of this study. W/e thank Dr F Klostermann for help with some of the clinical evaluations, and Dr Neil Scolding, Cambridge, UK, for helpful comments on the manuscript.
1 Prineas JW, Barnard RO, Kwon EE, et al. Multiple sclerosis: remyelination of nascent lesions. Ann Neurol 1993;33:13751.

2 Raine CS, Wu E. Multiple sclerosis: remyelination in acute lesions. F Neuropathol Exp Neurol 1993;52:199-204

3 Stangel M, Hartung H-P, Marx P, et al. Intravenous immunoglobulin treatment of neurological autoimmune diseases. F Neurol Sci 1998;153:203-14.

4 Fazekas F, Deisenhamer F, Strasser-Fuchs S, et al. Randomised placebo-controlled trial of monthly intravenous immunoglobulin therapy in relapsing-remitting multiple sclerosis. Lancet 1997;349:589-93.

5 Achiron A, Gabbay U, Gilad R, et al. Intravenous immunoglobulin treatment in multiple sclerosis: effect on relapses. Neurology 1998;50:398-402.

6 Sorensen PS, Wanscher B, Jensen CV, et al. Intravenous immunoglobulin G reduces MRI activity in relapsing multiple sclerosis. Neurology 1998;50:1273-81.

7 Rodriguez M, Lennon VA. Immunoglobulins promote remyelination in the central nervous system. Ann Neurol 1990;27:12-17

8 Rodriguez M, Miller DJ, Lennon VA. Immunoglobulins reactive with myelin basic protein promote CNS remyelination. Neurology 1996;46:538-45.

9 van Engelen BGM, Hommes OR, Pinckers A, et al. Improved vision after intravenous immunoglobulin in stable demyelinating optic neuritis. Ann Neurol 1992;32: $834-5$.

10 Noseworthy JH, Rodriguez M, An K-N, et al. IVIg treatment in multiple sclerosis: pilot study results and design of a placebo-controlled, double-blind clinical trial. Ann Neurol 1994;36:325.

11 Poser CM, Paty DW, Scheinberg L, et al. New diagnostic criteria for multiple sclerosis: guidelines for research protocols. Ann Neurol 1983;13:227-31

12 Kurtzke JF. Rating neurologic impairment in multiple sclerosis: an expanded disability status scale (EDSS). Neurology 1983;33:1444-52.

13 Hahn AF, Bolton CF, Zochodne D, et al. Intravenous immunoglobulin treatment in chronic inflammatory demyelinating polyneuropathy. A double-blind, placeboelinating polyneuropathy. A double-blind, place
controlled, cross-over study. Brain 1996;119:1067-77.

14 Hess CW. Die mittels Kortexreizung motorisch evozierten Potentiale (MEP). In: Stöhr M, Dichgens J, Diener HC, eds. Evozierte Potentiale. Berlin: Springer, 1989:589-634.

15 Young RR, Shahani BT. Clinical value and limitations of F-wave determination. Muscle Nerve 1978;1:248-9.

16 Ludolph AC, Wenning G, Masur H, et al. Die elektromagnetische Stimulation der Nervensystems. I. Normwerte im zentralen Nervensystem und Vergleich mit der elektrischen Stimulation. Zeitschrift EEG-EMG 1989;20:153-8.

17 Sipe JC, Knobler RL, Braheny SL, et al. A neurologic rating scale (NRS) for use in multiple sclerosis. Neurology 1984;34:1368-72.

18 Personius KE, Pandya S, King WM, et al. Facioscapulohumeral dystrophy natural history study: standardization of testing procedures and reliability of measurements. Phys Ther 1994;74:253-63.

19 Noseworthy JH, Weinshenker BG, O'Brien PC, et al. Intravenous immunoglobulin does not reverse recently acquired, apparently permanent weakness in multiple sclerosis. Ann Neurol 1997;42:421.

20 Noseworthy JH, O'Brien PC, Petterson TM, et al. Immunoglobulin administration does not reverse visual acuity in long-standing optic neuritis associated with multiple sclerosis. Ann Neurol 1998;44:504.

21 Asakura K, Miller DJ, Pease LR, et al. Targeting of IgMk antibodies to oligodendrocytes promotes CNS remyelination. F Neurosci 1998;18:7700-8.

22 Dwyer JM. Manipulating the immune system with immune globulins. N Engl f Med 1992;326:107-16.

23 Mouthon L, Kaveri SV, Spalter SH, et al. Mechanisms of action of intravenous immune globulin in immunemediated diseases. Clin Exp Immunol 1996;104(suppl 1):3-9. 